\title{
Bounds of the initial coefficient for sakaguchi function in the conical domain
}

\author{
S. Balaji ${ }^{1}$, B. Srutha Keerthi ${ }^{2}$ \\ ${ }^{1,2}$ Department of Mathematics, School of Advanced Sciences, VIT Chennai Campus
}

\section{Article Info}

Received May 27, 2019

\section{ABSTRACT}

In this paper, we consider a new class of sakaguchi type functions which is defined by Ruscheweyh q-differential operator. We investigated of coefficient inequalities and other interesting properties of this class.

\section{Keyword:}

Conical domain

Analytic functions

Sakaguchi type functions

k-uniformly starlike functions

q-differential Operator.

\section{Corresponding Author:}

\section{B. SruthaKeerthi}

Department of Mathematics, School of Advanced Sciences

VIT Chennai Campus, Chennai - 600 048, India

E-mail: sruthilaya06@yahoo.co.in

\section{Introduction}

Let $\mathcal{A}$ denotes the class of functions of the form

$$
\mathrm{f}(\mathrm{z})=\mathrm{z}+\sum_{\mathrm{n}=2}^{\infty} \mathrm{a}_{\mathrm{n}} \mathrm{z}^{\mathrm{n}}
$$

which are analytic in the open unit disk $D=\{z \in C:|z|<1\}$. If $f$ and $g$ are analytic function in $D$, then thefunction $\mathrm{f}$ is said to be subordinate to $\mathrm{g}$, and write $\mathrm{f}(\mathrm{z}) \prec \mathrm{g}(\mathrm{z})$, if there exists a function $\omega$ analytic in $\mathrm{D}$ with $\omega(0)=0$ and $|\omega(\mathrm{z})|<1$ for all $\mathrm{z} \in \mathrm{D}$, such thatf $(\mathrm{z})=\mathrm{g}(\omega(\mathrm{z})), \mathrm{z} \in \mathrm{D}$. Moreover, if the function $\mathrm{g}$ isunivalent in $D$, then $\mathrm{f}(\mathrm{z}) \prec \mathrm{g}(\mathrm{z})$ if and only if $\mathrm{f}(0)=\mathrm{g}(0)$ and $\mathrm{f}(\mathrm{D}) \subset \mathrm{g}(\mathrm{D})$.

Kanas and Wisniowka [5, 6], established the conic kind of domain $\Phi_{k}, \mathrm{k} \geq 0$ as

$$
\Phi_{\mathrm{k}}=\left\{\mathrm{u}+\mathrm{iv}: \mathrm{u}>k \sqrt{(\mathrm{u}-1)^{2}+\mathrm{v}^{2}}\right\}
$$

We note that $\Phi_{k}$ is a region in the right half-plane, symmetric with respect to real axis, and contains the point $(1,0)$. More precisely for $\mathrm{k}=0, \Phi_{0}$ is the right half-plane, for $0<\mathrm{k}<1, \Phi_{\mathrm{k}}$ is an unbounded region having boundary $\partial \Phi_{k}$, a rectangular hyperbola for $\mathrm{k}=1, \Phi_{1}$ is still an unbounded region where $\partial \Phi_{1}$ is a parabola, and for $\mathrm{k}>1, \Phi_{\mathrm{k}}$ is a bounded region enclosed by an ellipse. The extremalfunction for these conic regions are 


$$
\mathrm{p}_{k}(\mathrm{z})= \begin{cases}\frac{1+\mathrm{z}}{1-\mathrm{z}}, & \mathrm{k}=0 \\ 1+\frac{2}{\pi^{2}}\left(\log \frac{1+\sqrt{\mathrm{z}}}{1-\sqrt{\mathrm{z}}}\right)^{2}, & \mathrm{k}=1 \\ \frac{1}{1-\mathrm{k}^{2}} \cosh \left(\left(\frac{2}{\pi} \operatorname{arccosk}\right) \log \frac{1+\sqrt{\mathrm{z}}}{1-\sqrt{\mathrm{z}}}\right\}-\frac{k^{2}}{1-\mathrm{k}^{2}}, & 0<\mathrm{k}<1 \\ \frac{1}{\mathrm{k}^{2}-1} \sin \left(\frac{\pi}{2 \mathrm{k}(\mathrm{k \textrm {k }}} \int_{0}^{\frac{\mathrm{u}(\mathrm{z})}{\sqrt{\mathrm{k}}}} \frac{\mathrm{dk}}{\sqrt{1-\mathrm{t}^{2}} \sqrt{1-\mathrm{k}^{2} \mathrm{t}^{2}}}\right)+\frac{k^{2}}{\mathrm{k}^{2}-1}, & \mathrm{k}>1\end{cases}
$$

where $u(z)=\frac{2-\sqrt{k}}{1-\sqrt{\mathrm{kz}}}, \mathrm{z} \in \mathrm{D}$ and $\mathrm{k} \in(0,1)$ is chosen such that $\mathrm{k}=\cosh \left(\frac{\pi \mathrm{K}^{\prime}(\kappa)}{4 \mathrm{~K}(\kappa)}\right)$. Here $\mathrm{K}(\kappa)$ is Legendres's complete elliptic integral of first kind and $K^{\prime}(\kappa)=K\left(\sqrt{1-k^{2}}\right)$ and $K^{\prime}(t)$ is the complementary integral of $K(t)$ for details see $[1,5,6]$ and more recently $[9,12,14]$. If $P_{k}(z)=1+M_{1}(k) z+M_{2}(k) z^{2}+\cdots$, $\mathrm{z} \in \mathbb{D}$, then it was shown in [6] that for (2) one can have,

$\mathrm{M}_{2}(\mathrm{k})=\mathrm{E}(k) \mathrm{M}_{1}(\mathrm{k})$

$$
M_{1}(k)= \begin{cases}\frac{2 A^{2}}{1-k^{2}} & 0 \leq k<1 \\ \frac{8}{\pi^{2}} & k=1 \\ \frac{\pi^{2}}{4 K^{2}(t)^{2}(1+t) \sqrt{t}} & k>1\end{cases}
$$

where

$$
E(k)= \begin{cases}\frac{A^{2}+2}{3} & 0 \leq k<1 \\ \frac{\pi^{2}}{(4 K(t))^{2}\left(t^{2}+6 t+1\right)-\pi^{2}} & k=1 \\ 24 K(t)^{2}(1+t) \sqrt{t} & k>1\end{cases}
$$

with $\mathrm{A}=\frac{2}{\pi} \operatorname{arccosk}$

Further more a function $\mathrm{p}$ is said to be in the class $\mathrm{k}-\mathrm{P}[\mathrm{A}, \mathrm{B}]$ if and only if

$$
\mathrm{p}(\mathrm{z}) \prec \mathrm{q}_{\mathrm{k}}(\mathrm{z}), \quad \mathrm{k} \geq 0
$$

$$
\text { where } \mathrm{q}_{\mathrm{k}}(\mathrm{z})=\frac{(\mathrm{A}+1) \mathrm{p}_{\mathrm{k}}(\mathrm{z})-(\mathrm{A}-1)}{(\mathrm{B}+1) \mathrm{p}_{\mathrm{k}}(\mathrm{z})-(\mathrm{B}-1)}
$$

where $\mathrm{p}_{\mathrm{k}}$ is defined in (2) and $-1 \leq \mathrm{B}<\mathrm{A} \leq 1$. Geometrically the function $\mathrm{p} \in \mathrm{k}-\mathrm{P}[\mathrm{A}, \mathrm{B}]$ takes all the values from the domain $\Phi_{\mathrm{k}}[\mathrm{A}, \mathrm{B}],-1 \leq \mathrm{B}<\mathrm{A} \leq 1, \mathrm{k} \geq 0$, which is defined as:

$$
\begin{gathered}
\Phi_{k}[\mathrm{~A}, \mathrm{~B}]=\{\omega: \mathfrak{R}((\mathrm{c}(\omega))>\mathrm{k}|\mathrm{c}(\omega)|\} \\
\text { where } \quad \mathrm{c}\left(\omega \left(=\left(\frac{(\mathrm{B}-1) \omega-(\mathrm{A}-1)}{(\mathrm{B}+1) \omega-(\mathrm{A}+1)}\right)\right.\right.
\end{gathered}
$$

or equivalently $\Phi_{k}[A, B]$ is a set of numbers $\omega=u+i v$ such that

$$
\begin{aligned}
& {\left[\left(B^{2}-1\right)\left(u^{2}+v^{2}\right)-2(A B-1) u+\left(A^{2}-1\right)\right]^{2}} \\
& \quad>k\left[-2(B+1)\left(u^{2}+v^{2}\right)+2(A+B+2) u-2(A+1)^{2}+4(A-B)^{2} v^{2}\right]
\end{aligned}
$$

This domain represents the conic type of regions for detail see [11]. For any $n$ positive integer $n$, the q-integer number $n,[n, q]$ is defined by 


$$
[\mathrm{n}, \mathrm{q}]=\frac{1-\mathrm{q}^{\mathrm{n}}}{1-\mathrm{q}}=1+\mathrm{q}+\cdots+\mathrm{q}^{\mathrm{n}-1}[0, \mathrm{q}]=0, \quad \mathrm{q} \in(0,1)
$$

q-differential operator be defined by

$$
\partial_{\mathrm{q}} \mathrm{f}(\mathrm{z})=\frac{\mathrm{f}(\mathrm{qz})-\mathrm{f}(\mathrm{z})}{(\mathrm{q}-1) \mathrm{z}}, \quad(\mathrm{z} \in \mathrm{D})
$$

It is easy to observe that for $\mathrm{n} \in \mathrm{N}:=\{1,2,3 \ldots\}$ and $\mathrm{z} \in \mathrm{D}$

$$
\partial_{\mathrm{q}} \mathrm{z}^{\mathrm{n}}=[\mathrm{n}, \mathrm{q}] \mathrm{z}^{\mathrm{n}-1}
$$

Let the q-generated pochhammer symbol be defined as

$$
[\mathrm{r}, \mathrm{q}]_{\mathrm{n}}=[\mathrm{r}, \mathrm{q}][\mathrm{r}+1, \mathrm{q}][\mathrm{r}+2, \mathrm{q}] \ldots[\mathrm{r}+\mathrm{n}-1, \mathrm{q}]
$$

and for $r>0$ let the q-gamma function be defined as

$$
\Gamma_{\mathrm{q}}(\mathrm{r}+1)=[\mathrm{r}] \Gamma_{\mathrm{q}}(\mathrm{r}) \text { and } \Gamma_{\mathrm{q}}(1)=1
$$

These kind of operators see [2,3,13], play great in GFT. Kanas et al, defined Rucheweyh q-differential operator as follows:

Definition 1. [7]

For the function $\mathrm{f} \in \mathcal{A}$ is in the form (1), the Rucheweyh q-differential operator is:

$$
\mathrm{R}_{\mathrm{q}}^{\lambda} \mathrm{f}(\mathrm{z})=\mathrm{f}(\mathrm{z}) * \mathrm{~F}_{\mathrm{q}, \lambda+1}(\mathrm{z}), \quad(\mathrm{z} \in \mathrm{D}, \lambda>-1)
$$

where

$$
\begin{aligned}
\mathrm{F}_{\mathrm{q}, \lambda+1}(\mathrm{z})= & \mathrm{z}+\sum_{\mathrm{n}=2}^{\infty} \frac{\Gamma_{\mathrm{q}}(\mathrm{n}+\lambda)}{[\mathrm{n}-1, \mathrm{q}] ! \Gamma_{\mathrm{q}}(1+\lambda)} \mathrm{z}^{\mathrm{n}} \\
& =\mathrm{z}+\sum_{\mathrm{n}=2}^{\infty} \frac{[\lambda+1, \mathrm{q}]_{\mathrm{n}-1}}{[\mathrm{n}-1, \mathrm{q}] !} \mathrm{z}^{\mathrm{n}} \\
& =\mathrm{z}+\sum_{\mathrm{n}=2}^{\infty} \phi_{\mathrm{n}-1} \mathrm{z}^{\mathrm{n}}
\end{aligned}
$$

where

$$
\phi_{\mathrm{n}-1}=\frac{\Gamma_{\mathrm{q}}(\mathrm{n}+\lambda)}{[\mathrm{n}-1, \mathrm{q}] ! \Gamma_{\mathrm{q}}(1+\lambda)}=\frac{[\lambda+1, \mathrm{q}]_{\mathrm{n}-1}}{[\mathrm{n}-1, \mathrm{q}] !}
$$

from (7) we get that

$$
R_{q}^{0} f(z)=f(z), \quad R_{q}^{\prime} f(z)=z \partial_{q} f(z)
$$

and

$$
\mathrm{R}_{\mathrm{q}}^{\mathrm{m}} \mathrm{f}(\mathrm{z})=\frac{\mathrm{z} \partial_{\mathrm{q}}^{\mathrm{m}}\left(\mathrm{z}^{\mathrm{m}-1} \mathrm{f}(\mathrm{z})\right)}{[\mathrm{m}, \mathrm{q}] !} \quad(\mathrm{m} \in \mathrm{N})
$$

Using (7) and (8), the power series $R_{q}^{\lambda} f(z)$ is given by

$$
\begin{aligned}
\mathrm{R}_{\mathrm{q}}^{\lambda} \mathrm{f}(\mathrm{z})=\mathrm{z} & +\sum_{\mathrm{n}=2}^{\infty} \frac{\Gamma_{\mathrm{q}}(\mathrm{n}+\lambda)}{[\mathrm{n}-1, \mathrm{q}] ! \Gamma_{\mathrm{q}}(1+\lambda)} \mathrm{a}_{\mathrm{n}} \mathrm{z}^{\mathrm{n}} \\
& =\mathrm{z}+\sum_{\mathrm{n}=2}^{\infty} \frac{[\lambda+1, \mathrm{q}]_{\mathrm{n}-1}}{[\mathrm{n}-1, \mathrm{q}] !} \mathrm{a}_{\mathrm{n}} \mathrm{z}^{\mathrm{n}}
\end{aligned}
$$

Note that

$$
\lim _{\mathrm{q} \rightarrow 1} \Gamma_{\mathrm{q}, \lambda+1}(\mathrm{z})=\frac{\mathrm{z}}{(1-\mathrm{z})^{\lambda+1}}
$$

and

$$
\lim _{\mathrm{q} \rightarrow 1} \mathrm{R}_{\mathrm{q}}^{\lambda} \mathrm{f}(\mathrm{z})=\mathrm{f}(\mathrm{z}) * \frac{\mathrm{z}}{(1-\mathrm{z})^{\lambda+1}}
$$

When $\mathrm{q} \rightarrow 1$ see[16], we observe that 


$$
\mathrm{z} \partial\left(\mathrm{F}_{\mathrm{q}, \lambda+1}(\mathrm{z})\right)=\left(1+\frac{[\lambda, \mathrm{q}]}{\mathrm{q}^{\lambda}}\right) \mathrm{F}_{\mathrm{q}, \lambda+2}(\mathrm{z})-\frac{[\lambda, \mathrm{q}]}{\mathrm{q}^{\lambda}} \mathrm{F}_{\mathrm{q}, \lambda+1}(\mathrm{z})
$$

making use of (7), (10) and the properties of hadamard product we obtain the following equality

$$
\mathrm{z} \partial\left(\mathrm{R}_{\mathrm{q}}^{\lambda} \mathrm{f}(\mathrm{z})\right)=\left(1+\frac{[\lambda, \mathrm{q}]}{\mathrm{q}^{\lambda}}\right) \mathrm{R}_{\mathrm{q}}^{\lambda+1} \mathrm{f}(\mathrm{z})-\frac{[\lambda, \mathrm{q}]}{\mathrm{q}^{\lambda}} \mathrm{R}_{\mathrm{q}}^{\lambda} \mathrm{f}(\mathrm{z})
$$

If $\mathrm{q} \rightarrow 1$, the equality (11) implies

$$
z\left(R^{\lambda} f(z)\right)^{\prime}=(1+\lambda) R^{\lambda+1} f(z)-\lambda R^{\lambda} f(z)
$$

which is the familiar recurrent formula for the above operator. we now defined the following classes of functions.

\section{Definition 2.}

A function $\mathrm{f}(\mathrm{z}) \in \mathcal{A}$ is said to be in the class $\mathrm{k}-\mathrm{US}_{\mathrm{q}}(\lambda, \mathrm{A}, \mathrm{B}, \mathrm{t}), \mathrm{k} \geq 0, \beta \leq 0, \mathrm{t} \in \mathrm{C}$ with $|\mathrm{t}| \leq 1$ $-1 \leq \mathrm{B}<\mathrm{A} \leq 1$, if and only if

$$
\begin{array}{r}
\Re(c(X(z)))>k|c(X(z))| \\
X(z)=\frac{(1-t)\left(z_{\mathrm{q}} R_{\mathrm{q}}^{\lambda} \mathrm{f}(\mathrm{z})\right)}{\left(\mathrm{R}_{\mathrm{q}}^{\lambda} \mathrm{f}(\mathrm{z})-\mathrm{R}_{\mathrm{q}}^{\lambda} \mathrm{f}(\mathrm{tz})\right)}
\end{array}
$$

orequvalently,

$$
X(z)=\frac{(1-t)\left(z \partial_{q} R_{q}^{\lambda} f(z)\right)}{\left(R_{q}^{\lambda} f(z)-R_{q}^{\lambda} f(t z)\right)} \in k-P[A, B]
$$

\section{Definition 3.}

A function $\mathrm{f}(\mathrm{z}) \in \mathcal{A}$ is said to be in the class $\mathrm{k}-\mathrm{UC}_{\mathrm{q}}(\lambda, \mathrm{A}, \mathrm{B}, \mathrm{t}), \mathrm{k} \geq 0, \beta \leq 0, \mathrm{t} \in \mathrm{C}$ with $|\mathrm{t}| \leq 1,-1 \leq \mathrm{B}<\mathrm{A} \leq 1$, if and only if

$$
\mathrm{Y}(\mathrm{z})=\frac{\begin{array}{c}
\Re(\mathrm{c}(\mathrm{Y}(\mathrm{z})))>\mathrm{k}|\mathrm{c}(\mathrm{Y}(\mathrm{z}))| \\
(1-\mathrm{t})\left(\mathrm{z} \partial_{\mathrm{q}} \mathrm{R}_{\mathrm{q}}^{\lambda} \mathrm{f}(\mathrm{z})+\mathrm{z}^{2} \partial_{\mathrm{q}}^{2} \mathrm{R}_{\mathrm{q}}^{\lambda} \mathrm{f}(\mathrm{z})\right)
\end{array}}{\mathrm{z}\left(\partial_{\mathrm{q}} \mathrm{R}_{\mathrm{q}}^{\lambda} \mathrm{f}(\mathrm{z})-\mathrm{t} \partial_{\mathrm{q}} \mathrm{R}_{\mathrm{q}}^{\lambda} \mathrm{f}(\mathrm{tz})\right)}
$$

orequvalently,

$$
Y(z)=\frac{(1-t)\left(z \partial_{q} R_{q}^{\lambda} f(z)+z^{2} \partial_{q}^{2} R_{q}^{\lambda} f(z)\right)}{z\left(\partial_{q} R_{q}^{\lambda} f(z)-t \partial_{q} R_{q}^{\lambda} f(t z)\right)} \in k-U C_{q}
$$

\section{Definition 4.}

A function $\mathrm{f}(\mathrm{z}) \in \mathcal{A}$ is said to be in the class $\mathrm{k}-\mathrm{US}_{\mathrm{q}}(\lambda, \mathrm{A}, \mathrm{B}, \gamma, \mathrm{t}), \mathrm{k} \geq 0, \beta \leq 0, \mathrm{t} \in \mathrm{C}$ with $|\mathrm{t}| \leq 1$ $-1 \leq \mathrm{B}<\mathrm{A} \leq 1$, if and only if

$$
\begin{gathered}
\Re(c(G(z))>\mathrm{k}|c(G(z))| \\
\mathrm{G}(\mathrm{z})=\frac{(1-\mathrm{t})\left(\mathrm{z}_{\mathrm{q}} \mathrm{R}_{\mathrm{q}}^{\lambda} \mathrm{f}(\mathrm{z})+\gamma \mathrm{z}^{2} \partial_{\mathrm{q}}^{2} \mathrm{R}_{\mathrm{q}}^{\lambda} \mathrm{f}(\mathrm{z})\right)}{(1-\gamma)\left(\mathrm{R}_{\mathrm{q}}^{\lambda} \mathrm{f}(\mathrm{z})-\mathrm{R}_{\mathrm{q}}^{\lambda} \mathrm{f}(\mathrm{tz})\right)+\gamma \mathrm{z}\left(\partial_{\mathrm{q}} \mathrm{R}_{\mathrm{q}}^{\lambda} \mathrm{f}(\mathrm{z})-\mathrm{t} \partial_{\mathrm{q}} \mathrm{R}_{\mathrm{q}}^{\lambda} \mathrm{f}(\mathrm{tz})\right)}
\end{gathered}
$$

orequvalently,$$
G(z)=\frac{(1-t)\left(z_{q} R_{q}^{\lambda} f(z)+\gamma z^{2} \partial_{q}^{2} R_{q}^{\lambda} f(z)\right)}{(1-\gamma)\left(R_{q}^{\lambda} f(z)-R_{q}^{\lambda} f(t z)\right)+\gamma z\left(\partial_{q} R_{q}^{\lambda} f(z)-t \partial_{q} R_{q}^{\lambda} f(t z)\right)} \in k-P[A, B]
$$

\section{Remark 5.}

It is easily see that $\lim _{\mathrm{q} \rightarrow 1} \mathrm{k}-\mathrm{US}_{\mathrm{q}}(0, \mathrm{~A}, \mathrm{~B}, 0,0)=\mathrm{k}-\mathrm{ST}(\mathrm{A}, \mathrm{B})$ where $\mathrm{k}-\mathrm{ST}(\mathrm{A}, \mathrm{B})$ is a functions class, investigated by Noor and sarfraz [11] 


\section{Lemma 6.[15]}

Let $\mathrm{h}(\mathrm{z})=1+\sum_{\mathrm{n}=1}^{\infty} \mathrm{c}_{\mathrm{n}} \mathrm{z}^{\mathrm{n}}$ be subordinate to $\mathrm{H}(\mathrm{z})=1+\sum_{\mathrm{n}=1}^{\infty} \mathrm{C}_{\mathrm{n}} \mathrm{z}^{\mathrm{n}}$. If $\mathrm{H}(\mathrm{z})$ is univalent in $\mathrm{D}$ and $\mathrm{H}(\mathrm{E})$ is convex, then

$$
\left|\mathrm{c}_{\mathrm{n}}\right| \leq\left|\mathrm{C}_{1}\right|, \mathrm{n} \geq 1
$$

Lemma 7. $[8,10]$

If $\mathrm{q}(\mathrm{z})=1+\mathrm{c}_{1} \mathrm{z}+\mathrm{c}_{2} \mathrm{z}^{2}+\cdots$ is an analytic function with positive real part in $\mathrm{D}$ then,

$$
\left|\mathrm{c}_{2}-\mathrm{vc} \mathrm{c}_{1}^{2}\right| \leq 2 \max \{1,2 \mathrm{v}-1 \mid\}
$$

The result is sharp for the function

$$
\mathrm{q}(\mathrm{z})=\frac{1+\mathrm{z}^{2}}{1-\mathrm{z}^{2}} \quad(\text { or }) \quad \mathrm{q}(\mathrm{z})=\frac{1+\mathrm{z}}{1-\mathrm{z}}
$$

Lemma 8. [8]

If the function $\omega \in \mathrm{D}$ is in the form

Then,

$$
\omega(\mathrm{z})=\mathrm{c}_{1} \mathrm{z}+\mathrm{c}_{2} \mathrm{z}^{2}+\cdots \mathrm{z} \in \mathrm{D}
$$

$$
\left|\mathrm{c}_{2}-\mathrm{vc}_{1}^{2}\right| \leq 1+(|\mathrm{v}|-1)\left|\mathrm{c}_{1}\right|^{2}
$$

where $\mathrm{v}$ is the complex number

Lemma 9. [11]

Let $\mathrm{k} \in[0, \infty)$ be fixed and $\mathrm{q}_{\mathrm{k}}(\mathrm{z})$ in the form (5)then

$$
\mathrm{q}_{\mathrm{k}}(\mathrm{z})=1+\mathrm{H}_{1}(\mathrm{k}) \mathrm{z}+\mathrm{H}_{2}(\mathrm{k}) \mathrm{z}^{2}+\cdots, \mathrm{z} \in \mathrm{D}
$$

and

$$
\begin{aligned}
& \mathrm{H}_{1}: \mathrm{H}_{1}(\mathrm{k})=\frac{\mathrm{A}-\mathrm{B}}{2} \mathrm{M}_{1}(\mathrm{k}) \\
& \mathrm{H}_{2}: \mathrm{H}_{2}(\mathrm{k})=\frac{\mathrm{A}-\mathrm{B}}{4}\left\{2 \mathrm{E}(\mathrm{k})-(\mathrm{B}+1) \mathrm{H}_{1}\right\} \mathrm{M}_{1}(\mathrm{k})
\end{aligned}
$$

where $\mathrm{M}_{1}(\mathrm{k})$ and $\mathrm{E}(\mathrm{k})$ are defined in (3) and (4)

\section{Main Results}

\section{Theorem 10:}

A function $\mathrm{f} \in \mathcal{A}$ and of the form (1) is in the class $\mathrm{k}-\mathrm{US}_{\mathrm{q}}(\lambda, \mathrm{A}, \mathrm{B}, \gamma, \mathrm{t})$, if it satisfies the condition

$$
\begin{gathered}
\sum_{n=2}^{\infty}\left\{2(k+1)\left\{(1-\gamma) u_{n}+\left(\gamma u_{n}-1\right)[n, q]-\gamma[n, q][n-1, q]\right\}+\mid(B+1)\{[n, q]+\gamma[n, q][n-1, q]\}\right. \\
\left.-(A+1)\left\{(1-\gamma) u_{n}+\gamma[n, q] u_{n}\right\} \mid\right\} \phi_{n-1}\left|a_{n}\right| \leq|B-A|
\end{gathered}
$$

roof.

Assume (13) is hold, then it suffices to show that 


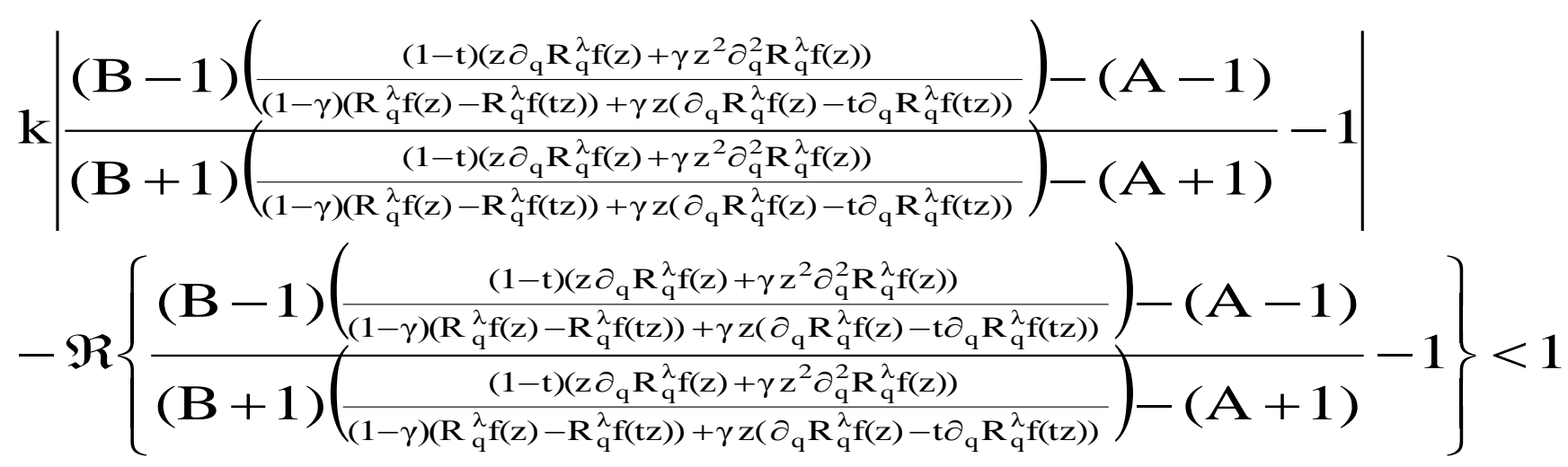

we have

$$
\begin{aligned}
& k\left|\frac{(B-1)\left(\frac{(1-t)\left(z \partial_{q} R_{q}^{\lambda} f(z)+\gamma z^{2} \partial_{q}^{2} R_{q}^{\lambda} f(z)\right)}{(1-\gamma)\left(R_{q}^{\lambda} f(z)-R_{q}^{\lambda} f(t z)\right)+\gamma z\left(\partial_{q} R_{q}^{\lambda} f(z)-t \partial_{q} R_{q}^{\lambda} f(t z)\right)}\right)-(A-1)}{(B+1)\left(\frac{(1-t)\left(z \partial_{q} R_{q}^{\lambda} f(z)+\gamma z^{2} \partial_{q}^{2} R_{q}^{\lambda} f(z)\right)}{(1-\gamma)\left(R_{q}^{\lambda} f(z)-R_{q}^{\lambda} f(t z)\right)+\gamma z\left(\partial_{q} R_{q}^{\lambda} f(z)-t \partial_{q} R_{q}^{\lambda} f(t z)\right)}\right)-(A+1)}-1\right| \\
& -\mathfrak{R}\left\{\frac{(B-1)\left(\frac{(1-t)\left(z \partial_{\mathrm{q}} \mathrm{R}_{\mathrm{q}}^{\lambda} \mathrm{f}(\mathrm{z})+\gamma \mathrm{z}^{2} \partial_{\mathrm{q}}^{2} \mathrm{R}_{\mathrm{q}}^{\lambda} \mathrm{f}(\mathrm{z})\right)}{(1-\gamma)\left(\mathrm{R}_{\mathrm{q}}^{\lambda} \mathrm{f}(\mathrm{z})-\mathrm{R}_{\mathrm{q}}^{\lambda} \mathrm{f}(\mathrm{tz})\right)+\gamma \mathrm{z}\left(\partial_{\mathrm{q}} \mathrm{R}_{\mathrm{q}}^{\lambda} \mathrm{f}(\mathrm{z})-\mathrm{t} \partial_{\mathrm{q}} \mathrm{R}_{\mathrm{q}}^{\lambda} \mathrm{f}(\mathrm{tz})\right)}\right)-(\mathrm{A}-1)}{(\mathrm{B}+1)\left(\frac{(1-t)\left(\mathrm{z} \partial_{\mathrm{q}} \mathrm{R}_{\mathrm{q}}^{\lambda} \mathrm{f}(\mathrm{z})+\gamma \mathrm{z}^{2} \partial_{\mathrm{q}}^{2} \mathrm{R}_{\mathrm{q}}^{\lambda} \mathrm{f}(\mathrm{z})\right)}{(1-\gamma)\left(\mathrm{R}_{\mathrm{q}}^{\lambda} \mathrm{f}(\mathrm{z})-\mathrm{R}_{\mathrm{q}}^{\lambda} \mathrm{f}(\mathrm{tz})\right)+\gamma \mathrm{z}\left(\partial_{\mathrm{q}} \mathrm{R}_{\mathrm{q}}^{\lambda} \mathrm{f}(\mathrm{z})-\mathrm{t} \partial_{\mathrm{q}} \mathrm{R}_{\mathrm{q}}^{\lambda} \mathrm{f}(\mathrm{tz})\right)}\right)-(\mathrm{A}+1)}-1\right\} \\
& \leq(k+1)\left|\frac{(B-1)(1-t)\left(z_{q} R_{q}^{\lambda} f(z)+\gamma z^{2} \partial_{q}^{2} R_{q}^{\lambda} f(z)\right)-(A-1)\left[(1-\gamma)\left(R_{q}^{\lambda} f(z)-R_{q}^{\lambda} f(t z)\right)+\gamma z\left(\partial_{q} R_{q}^{\lambda} f(z)-t \partial_{q} R_{q}^{\lambda} f(t z)\right)\right]}{(B+1)(1-t)\left(z_{q} R_{q}^{\lambda} f(z)+\gamma z^{2} \partial_{q}^{2} R_{q}^{\lambda} f(z)\right)-(A+1)\left[(1-\gamma)\left(R_{q}^{\lambda} f(z)-R_{q}^{\lambda} f(t z)\right)+\gamma z\left(\partial_{q} R_{q}^{\lambda} f(z)-t \partial_{q} R_{q}^{\lambda} f(t z)\right)\right]}\right| \\
& =2(k+1)\left|\frac{(1-\gamma)\left(R_{q}^{\lambda} f(z)-R_{q}^{\lambda} f(t z)\right)+\gamma z\left(\partial_{q} R_{q}^{\lambda} f(z)-t \partial_{q} R_{q}^{\lambda} f(t z)\right)-(1-t)\left(z \partial_{q} R_{q}^{\lambda} f(z)+\gamma z^{2} \partial_{q}^{2} R_{q}^{\lambda} f(z)\right)}{(B+1)(1-t)\left(z \partial_{q} R_{q}^{\lambda} f(z)+\gamma z^{2} \partial_{q}^{2} R_{q}^{\lambda} f(z)\right)-(A+1)\left[(1-\gamma)\left(R_{q}^{\lambda} f(z)-R_{q}^{\lambda} f(t z)\right)+\gamma z\left(\partial_{q} R_{q}^{\lambda} f(z)-t \partial_{q} R_{q}^{\lambda} f(t z)\right)\right.}\right| \\
& =2(k+1)\left|\frac{\sum_{n=2}^{\infty}\left\{(1-\gamma) u_{n}+\left(\gamma u_{n}-1\right)[n, q]-\gamma[n, q][n-1, q]\right\} \phi_{n-1} a_{n} z^{n}}{(B-A) z+\sum_{n=2}^{\infty}\left\{(B+1)\{[n, q]+\gamma[n, q][n-1, q]\}-(A+1)\left\{(1-\gamma) u_{n}+\gamma[n, q] u_{n}\right\}\right\} \phi_{n-1} a_{n} z^{n}}\right| \\
& =2(\mathrm{k}+1) \frac{\sum_{\mathrm{n}=2}^{\infty}\left\{(1-\gamma) \mathrm{u}_{\mathrm{n}}+\left(\gamma \mathrm{u}_{\mathrm{n}}-1\right)[\mathrm{n}, \mathrm{q}]-\gamma[\mathrm{n}, \mathrm{q}][\mathrm{n}-1, \mathrm{q}]\right\} \phi_{\mathrm{n}-1}\left|\mathrm{a}_{\mathrm{n}}\right|}{|\mathrm{B}-\mathrm{A}|-\sum_{\mathrm{n}=2}^{\infty}\left\{(\mathrm{B}+1)\{[\mathrm{n}, \mathrm{q}]+\gamma[\mathrm{n}, \mathrm{q}][\mathrm{n}-1, \mathrm{q}]\}-(\mathrm{A}+1)\left\{(1-\gamma) \mathrm{u}_{\mathrm{n}}+\gamma[\mathrm{n}, \mathrm{q}] \mathrm{u}_{\mathrm{n}}\right\}\right\} \phi_{\mathrm{n}-1}\left|\mathrm{a}_{\mathrm{n}}\right|}
\end{aligned}
$$

$<1$ by (13)

When $\mathrm{q} \rightarrow 1$ and $\gamma=0$ we have,

\section{Corollary 11.}

A function $\mathrm{f} \in \mathrm{A}$ and of the form (1) is in the class $\mathrm{k}-\mathrm{US}_{\mathrm{q}}(\lambda, \mathrm{A}, \mathrm{B}, \mathrm{t})$, if it satisfies the condition,

$$
\sum_{n=2}^{\infty}\left\{2(\mathrm{k}+1)\left(\mathrm{u}_{\mathrm{n}}-\mathrm{n}\right)+\left|\mathrm{n}(\mathrm{B}+1)-\mathrm{u}_{\mathrm{n}}(\mathrm{A}+1)\right|\right\} \phi_{\mathrm{n}-1}\left|\mathrm{a}_{\mathrm{n}}\right| \leq|\mathrm{B}-\mathrm{A}|
$$

When $\mathrm{q} \rightarrow 1$ and $\gamma=1$ we have,

\section{Corollary 12.}

A function $\mathrm{f} \in \mathrm{A}$ and of the form (1) is in the class $\mathrm{k}-\mathrm{UC}_{\mathrm{q}}(\lambda, \mathrm{A}, \mathrm{B}, \mathrm{t})$, if it satisfies the condition, 


$$
\sum_{n=2}^{\infty}\left\{2(k+1)\left\{n\left(u_{n}-1\right)-n(n-1)\right\}+\left|(B+1) n^{2}-n_{n}(A+1)\right|\right\} \phi_{n-1}\left|a_{n}\right| \leq|B-A|
$$

\section{Theorem 13.}

If $\mathrm{f}(\mathrm{z}) \in \mathrm{k}-\mathrm{US}_{\mathrm{q}}(\lambda, \mathrm{A}, \mathrm{B}, \gamma, \mathrm{t})$, and is of the form (1). then

$$
\left|a_{n}\right| \leq \prod_{j=0}^{n-2}\left\{\frac{\left|M_{1}(A-B) u_{j+1}\{(1-\gamma)+\gamma[j+1, q]\}-2\left[\{1+\gamma[j, q]\}[j+1, q]-\{(1-\gamma)+\gamma[j+1, q]\} u_{j+1}\right] B\right|}{2\left[\{1+\gamma[j+1, q]\}[j+2, q]-\{(1-\gamma)+\gamma[j+2, q]\} u_{j+2}\right] \phi_{n-1}}\right\}, n \geq 2
$$

where $M_{1}(k)$ is defined by (3)

\section{Proof.}

Let

$$
\frac{(1-t)\left(z_{q} R_{q}^{\lambda} f(z)+\gamma z^{2} \partial_{q}^{2} R_{q}^{\lambda} f(z)\right)}{(1-\gamma)\left(R_{q}^{\lambda} f(z)-R_{q}^{\lambda} f(t z)\right)+\gamma z\left(\partial_{q} R_{q}^{\lambda} f(z)-t \partial_{q} R_{q}^{\lambda} f(t z)\right)}=p(z)
$$

then

$$
\begin{aligned}
\mathrm{p}(\mathrm{z}) & \prec \mathrm{q}_{\mathrm{k}}(\mathrm{z}) \\
& =\left[(\mathrm{A}+1) \mathrm{p}_{\mathrm{k}}(\mathrm{z})-(\mathrm{A}-1)\right]\left[(\mathrm{B}+1) \mathrm{p}_{\mathrm{k}}(\mathrm{z})-(\mathrm{B}-1)\right]^{-1} \\
& =\frac{(\mathrm{A}-1)}{(\mathrm{B}-1)}+\left(\frac{(\mathrm{A}-1)(\mathrm{B}+1)}{(\mathrm{B}-1)^{2}}-\frac{(\mathrm{A}+1)}{(\mathrm{B}-1)}\right)\left(\mathrm{p}_{\mathrm{k}}(\mathrm{z})\right)+\left(\frac{(\mathrm{A}-1)(\mathrm{B}+1)^{2}}{(\mathrm{~B}-1)^{3}}-\frac{(\mathrm{A}+1)(\mathrm{B}+1)}{(\mathrm{B}-1)^{2}}\right)\left(\mathrm{p}_{\mathrm{k}}(\mathrm{z})\right)^{2}+\cdots
\end{aligned}
$$

By taking $\mathrm{p}_{\mathrm{k}}(\mathrm{z})=1+\mathrm{M}_{1}(\mathrm{k}) \mathrm{z}+\mathrm{M}_{2}(\mathrm{k}) \mathrm{z}^{2}+\cdots$

after some simplification, we obtain,

$$
\mathrm{p}(\mathrm{z}) \prec \sum_{\mathrm{n}=1}^{\infty} \frac{-2(\mathrm{~B}+1)^{\mathrm{n}-1}}{(\mathrm{~B}-1)^{\mathrm{n}}}+\left\{\sum_{\mathrm{n}=1}^{\infty} \frac{-2 \mathrm{n}(\mathrm{A}-\mathrm{B})(\mathrm{B}+1)^{\mathrm{n}-1}}{(\mathrm{~B}-1)^{\mathrm{n}+1}}\right\} \mathbf{M}_{1}(\mathrm{k})+\cdots
$$

Now we see that the series $\sum_{n=1}^{\infty} \frac{-2(B+1)^{n-1}}{(B-1)^{n}}$ and $\sum_{n=1}^{\infty} \frac{-2 n(A-B)(B+1)^{n-1}}{(B-1)^{n+1}}$ are convergent and converge to 1 and $\frac{\mathrm{A}-\mathrm{B}}{2}$ respectively. Therefore,

$\mathrm{p}(\mathrm{z}) \prec 1+\frac{\mathrm{A}-\mathrm{B}}{2} \mathrm{M}_{1}(\mathrm{k}) \mathrm{z}+\cdots$

Now if $\mathrm{p}(\mathrm{z})=1+\sum_{\mathrm{n}=1}^{\infty} \mathrm{c}_{\mathrm{n}} \mathrm{z}^{\mathrm{n}}$, then by lemma [6], we have

$$
\left|c_{n}\right| \leq \frac{A-B}{2} M_{1}(k), \quad n \geq 1
$$

Now from (15) we have

$$
(1-t)\left[z \partial_{q} R_{q}^{\lambda} f(z)+\gamma z^{2} \partial_{q}^{2} R_{q}^{\lambda} f(z)\right]=\left[(1-\gamma)\left(R_{q}^{\lambda} f(z)-R_{q}^{\lambda} f(t z)\right)+\gamma z\left(\partial_{q} R_{q}^{\lambda} f(z)-t \partial_{q} R_{q}^{\lambda} f(t z)\right)\right] p(\not)
$$

by simple calculation provides us,

$$
\begin{gathered}
\left|a_{n}\right| \leq \frac{\sum_{j=1}^{n-1}[(1-\gamma)+\gamma[j, q]] u_{j} \phi_{j-1}\left|a_{j}\right|\left|c_{n-j}\right|}{\left[\{1+\gamma[n-1, q]\}[n, q]-\{(1-\gamma)+\gamma[n, q]\} u_{n}\right] \phi_{n-1}}, \quad a_{1}=1 \\
\left|a_{n}\right| \leq \frac{(A-B)\left|M_{1}(k)\right| \sum_{j=1}^{n-1}[(1-\gamma)+\gamma[j, q]] u_{j} \phi_{j-1}\left|a_{j}\right|}{2\left[\{1+\gamma[n-1, q]\}[n, q]-\{(1-\gamma)+\gamma[n, q]\} u_{n}\right] \phi_{n-1}}, \quad a_{1}=1
\end{gathered}
$$

Now we prove that 


$$
\begin{aligned}
& \frac{(A-B)\left|M_{1}(k)\right| \sum_{j=1}^{n-1}[(1-\gamma)+\gamma[j, q]] u_{j} \phi_{j-1}\left|a_{j}\right|}{2\left[\{1+\gamma[n-1, q]\}[n, q]-\{(1-\gamma)+\gamma[n, q]\} u_{n}\right] \phi_{n-1}} \\
& \leq \prod_{j=0}^{n-2}\left\{\frac{\left|M_{1}(k)(A-B) u_{j+1}\{(1-\gamma)+\gamma[j+1, q]\}-2\left[\{1+\gamma[j, q]\}[j+1, q]-\{(1-\gamma)+\gamma[j+1, q]\} u_{j+1}\right] B\right|}{2\left[\{1+\gamma[j+1, q]\}[j+2, q]-\{(1-\gamma)+\gamma[j+2, q]\} u_{j+2}\right] \phi_{n-1}}\right\}
\end{aligned}
$$

For this we use the induction method

For $n=2$ From (17), we have

$$
\left|\mathrm{a}_{2}\right| \leq \frac{(\mathrm{A}-\mathrm{B})\left|\mathrm{M}_{1}(\mathrm{k})\right|}{2\left[(1+\gamma)[2, \mathrm{q}]-\{(1-\gamma)+\gamma[2, \mathrm{q}]\} \mathrm{u}_{2}\right] \phi_{1}}
$$

From (14) we have

$$
\left|\mathrm{a}_{2}\right| \leq \frac{(\mathrm{A}-\mathrm{B})\left|\mathrm{M}_{1}(\mathrm{k})\right|}{2\left[(1+\gamma)[2, \mathrm{q}]-\{(1-\gamma)+\gamma[2, \mathrm{q}]\} \mathrm{u}_{2}\right] \phi_{1}}
$$

For $\mathrm{n}=3$ From (17) we have

$$
\begin{aligned}
\left|\mathrm{a}_{3}\right| \leq & \frac{(\mathrm{A}-\mathrm{B})\left|\mathbf{M}_{1}\right|}{2\left[\{1+\gamma[2, \mathrm{q}]\}[3, \mathrm{q}]-\{(1-\gamma+\gamma[3, \mathrm{q}])\} \mathrm{u}_{3}\right] \phi_{2}} \\
& \left(\frac{\left|\mathbf{M}_{1}(\mathrm{k})\right|(\mathrm{A}-\mathrm{B}) \mathrm{u}_{2}[(1-\gamma)+\gamma[2, \mathrm{q}]]+2\left[(1+\gamma)[2, \mathrm{q}]-\{(1-\gamma)+\gamma[2, \mathrm{q}]\} \mathrm{u}_{2}\right]}{2\left[(1+\gamma)[2, \mathrm{q}]-\{(1-\gamma)+\gamma[2, \mathrm{q}]\} \mathrm{u}_{2}\right]}\right)
\end{aligned}
$$

from (14) we have

$$
\begin{aligned}
\left|\mathrm{a}_{3}\right| \leq & \frac{(\mathrm{A}-\mathrm{B})\left|\mathrm{M}_{1}\right|}{2\left[(1+\gamma)[2, \mathrm{q}]-\{(1-\gamma)+\gamma[2, \mathrm{q}]\} \mathrm{u}_{2}\right] \phi_{2}} \\
& \left(\frac{\left|\mathrm{M}_{1}(\mathrm{k})\right|(\mathrm{A}-\mathrm{B}) \mathrm{u}_{2}[(1-\gamma)+\gamma[2, \mathrm{q}]]+2\left[(1+\gamma)[2, \mathrm{q}]-\{(1-\gamma)+\gamma[2, \mathrm{q}]\} \mathrm{u}_{2}\right]}{2\left[\{1+\gamma[2, \mathrm{q}]\}[3, \mathrm{q}]-\{(1-\gamma)+\gamma[3, \mathrm{q}]\} \mathrm{u}_{3}\right]}\right)
\end{aligned}
$$

Let the hypothesis be true for $\mathrm{n}=\mathrm{m}$ from (16) we have,

$$
\left|\mathbf{a}_{\mathrm{m}}\right| \leq \frac{(\mathrm{A}-\mathrm{B})\left|\mathbf{M}_{1}(\mathrm{k})\right| \sum_{\mathrm{j}=1}^{\mathrm{m}-1}[(1-\gamma)+\gamma[\mathrm{j}, \mathrm{q}]] \mathrm{u}_{\mathrm{j}} \phi_{\mathrm{j}-1}\left|\mathrm{a}_{\mathrm{j}}\right|}{2\left[\{1+\gamma[\mathrm{m}-1, \mathrm{q}]\}[\mathrm{m}, \mathrm{q}]-\{(1-\gamma)+\gamma[\mathrm{m}, \mathrm{q}]\} \mathrm{u}_{\mathrm{m}}\right] \phi_{\mathrm{m}-1}}, \quad \mathbf{a}_{1}=1
$$

From(14) we have

$$
\left|a_{m}\right| \leq \prod_{j=0}^{m-2}\left\{\frac{\left|\mathbf{M}_{1}\right|(A-B) u_{j+1}\{(1-\gamma)+\gamma[j+1, q]\}+2\left[\{1+\gamma[j, q]\}[j+1, q]-\{(1-\gamma)+\gamma[j+1, q]\} u_{j+1}\right]}{2\left[\{1+\gamma[j+1, q]\}[j+2, q]-\{(1-\gamma)+\gamma[j+2, q]\} u_{j+2}\right] \phi_{m-1}}\right\}
$$

By induction hypothesis, we have

$$
\begin{aligned}
& \frac{(A-B)\left|M_{1}(k)\right| \sum_{j=1}^{m-1}[(1-\gamma)+\gamma[j, q]] u_{j} \phi_{j-1}\left|a_{j}\right|}{2\left[\{1+\gamma[m-1, q]\}[m, q]-\{(1-\gamma)+\gamma[m, q]\} u_{m}\right] \phi_{m-1}} \\
& \leq \prod_{j=0}^{m-2}\left\{\frac{\left|M_{1}\right|(A-B) u_{j+1}\{(1-\gamma)+\gamma[j+1, q]\}+2\left[\{1+\gamma[j, q]\}[j+1, q]-\{(1-\gamma)+\gamma[j+1, q]\} u_{j+1}\right]}{2\left[\{1+\gamma[j+1, q]\}[j+2, q]-\{(1-\gamma)+\gamma[j+2, q]\} u_{j+2}\right] \phi_{m-1}}\right\}
\end{aligned}
$$

Multiplying both sides (19) by

$$
\frac{\left|\mathrm{M}_{1}\right|(\mathrm{A}-\mathrm{B}) \mathrm{u}_{\mathrm{m}}\{(1-\gamma)+\gamma[\mathrm{m}, \mathrm{q}]\}+2\left[\{1+\gamma[\mathrm{m}-1, \mathrm{q}]\}[\mathrm{m}, \mathrm{q}]-\{(1-\gamma)+\gamma[\mathrm{m}, \mathrm{q}]\} \mathrm{u}_{\mathrm{m}}\right]}{2\left[\{1+\gamma[\mathrm{m}, \mathrm{q}]\}[\mathrm{m}+1, \mathrm{q}]-\{(1-\gamma)+\gamma[\mathrm{m}+1, \mathrm{q}]\} \mathrm{u}_{\mathrm{m}+1}\right]}
$$

we have

$$
\prod_{j=0}^{m-2}\left\{\frac{\left|M_{1}\right|(A-B) u_{j+1}\{(1-\gamma)+\gamma[j+1, q]\}+2\left[\{1+\gamma[j, q]\}[j+1, q]-\{(1-\gamma)+\gamma[j+1, q]\} u_{j+1}\right]}{2\left[\{1+\gamma[j+1, q]\}[j+2, q]-\{(1-\gamma)+\gamma[j+2, q]\} u_{j+2}\right] \phi_{m-1}}\right\}
$$




$$
\begin{aligned}
& \geq \frac{\left|\mathbf{M}_{1}\right|(\mathrm{A}-\mathrm{B}) \mathrm{u}_{\mathrm{m}}\{(1-\gamma)+\gamma[\mathrm{m}, \mathrm{q}]\}+2\left[\{1+\gamma[\mathrm{m}-1, \mathrm{q}]\}[\mathrm{m}, \mathrm{q}]-\{(1-\gamma)+\gamma[\mathrm{m}, \mathrm{q}]\} \mathrm{u}_{\mathrm{m}}\right]}{2\left[\{1+\gamma[\mathrm{m}, \mathrm{q}]\}[\mathrm{m}+1, \mathrm{q}]-\{(1-\gamma)+\gamma[\mathrm{m}+1, \mathrm{q}]\} \mathrm{u}_{\mathrm{m}+1}\right]} \\
& \quad \times\left\{\left|\mathrm{a}_{\mathrm{m}}\right| \leq \frac{(\mathrm{A}-\mathrm{B})\left|\mathbf{M}_{1}(\mathrm{k})\right| \sum_{\mathrm{j}=1}^{\mathrm{m}-1}[(1-\gamma)+\gamma[\mathrm{j}, \mathrm{q}]] \mathrm{u}_{\mathrm{j}} \phi_{\mathrm{j}-1}\left|\mathrm{a}_{\mathrm{j}}\right|}{2\left[\{1+\gamma[\mathrm{m}-1, \mathrm{q}]\}[\mathrm{m}, \mathrm{q}]-\{(1-\gamma)+\gamma[\mathrm{m}, \mathrm{q}]\} \mathrm{u}_{\mathrm{m}}\right] \phi_{\mathrm{m}-1}}\right\} \\
& =\frac{(\mathrm{A}-\mathrm{B})\left|\mathrm{M}_{1}\right|}{2\left[\{1+\gamma[\mathrm{m}, \mathrm{q}]\}[\mathrm{m}+1, \mathrm{q}]-\{(1-\gamma)+\gamma[\mathrm{m}+1, \mathrm{q}]\} \mathrm{u}_{\mathrm{m}+1}\right] \phi_{\mathrm{m}-1}} \\
& =\frac{\left[\sum_{\mathrm{j}=1}^{\mathrm{m}-1}[(1-\gamma)+\gamma[\mathrm{j}, \mathrm{q}]] \phi_{\mathrm{j}-1}\left|\mathrm{a}_{\mathrm{j}}\right|+\mathrm{u}_{\mathrm{m}}\{(1-\gamma)+\gamma[\mathrm{m}, \mathrm{q}]\} \phi_{\mathrm{m}-1}\left|\mathrm{a}_{\mathrm{m}}\right|\right]}{2\left[\{1+\gamma[\mathrm{m}, \mathrm{q}]\}[\mathrm{m}+1, \mathrm{q}]-\{(1-\gamma)+\gamma[\mathrm{m}+1, \mathrm{q}]\} \mathrm{u}_{\mathrm{m}+1}\right] \phi_{\mathrm{m}-1}} \\
& \quad \times \sum_{\mathrm{j}=1}^{\mathrm{m}}[(1-\gamma)+\gamma[\mathrm{j}, \mathrm{q}]] \phi_{\mathrm{j}-1}\left|\mathrm{a}_{\mathrm{j}}\right|
\end{aligned}
$$

That is

$$
\begin{aligned}
& \frac{(\mathrm{A}-\mathrm{B})\left|\mathrm{M}_{1}(\mathrm{k})\right| \sum_{\mathrm{j}=1}^{\mathrm{m}-1}[(1-\gamma)+\gamma[\mathrm{j}, \mathrm{q}]] \mathrm{u}_{\mathrm{j}} \phi_{\mathrm{j}-1}\left|\mathrm{a}_{\mathrm{j}}\right|}{2\left[\{1+\gamma[\mathrm{m}, \mathrm{q}]\}[\mathrm{m}+1, \mathrm{q}]-\{(1-\gamma)+\gamma[\mathrm{m}+1, \mathrm{q}]\} \mathrm{u}_{\mathrm{m}+1}\right] \phi_{\mathrm{m}-1}} \\
& \leq \prod_{\mathrm{j}=0}^{\mathrm{m}-2}\left\{\frac{\left|\mathrm{M}_{1}\right|(\mathrm{A}-\mathrm{B}) \mathrm{u}_{\mathrm{j}+1}\{(1-\gamma)+\gamma[\mathrm{j}+1, \mathrm{q}]\}+2\left[\{1+\gamma[\mathrm{j}, \mathrm{q}]\}[\mathrm{j}+1, \mathrm{q}]-\{(1-\gamma)+\gamma[\mathrm{j}+1, \mathrm{q}]\} \mathrm{u}_{\mathrm{j}+1}\right]}{2\left[\{1+\gamma[\mathrm{j}+1, \mathrm{q}]\}[\mathrm{j}+2, \mathrm{q}]-\{(1-\gamma)+\gamma[\mathrm{j}+2, \mathrm{q}]\} \mathrm{u}_{\mathrm{j}+2}\right] \phi_{\mathrm{m}-1}}\right\}
\end{aligned}
$$

Which gives (19).

When $\mathrm{q} \rightarrow 1$ and $\gamma=0$ we have,

\section{Corollary 14.}

A function $\mathrm{f} \in \mathrm{A}$ and of the form (1) is in $\mathrm{k}-\mathrm{US}_{\mathrm{q}}(\lambda, \mathrm{A}, \mathrm{B}, \mathrm{t})$, if,

is satisfied.

$$
\left|a_{n}\right| \leq \prod_{j=0}^{n-2}\left(\frac{\left|M_{1}(k)(A-B) u_{j+1}-2\left[j+1-u_{j+1}\right] B\right|}{2\left[j+2-u_{j+2}\right] \phi_{n-1}}\right)
$$

When $\mathrm{q} \rightarrow 1$ and $\gamma=1$ we have,

\section{Corollary 15.}

A function $\mathrm{f} \in \mathrm{A}$ and of the form (1) is said to be in the class $\mathrm{k}-\mathrm{UC}_{\mathrm{q}}(\lambda, \mathrm{A}, \mathrm{B}, \mathrm{t})$, if,

is true.

$$
\left|a_{n}\right| \leq \prod_{j=0}^{n-2}\left(\frac{\left|M_{1}(k)(A-B)(j+1)-2(j+1)\left[1+j-u_{j+1}\right] B\right|}{2(j+2)\left[2+j-u_{j+2}\right] \phi_{n-1}}\right)
$$

Theorem 16.

Let $-1 \leq \mathrm{B}<\mathrm{A} \leq 1$ and $\mathrm{t}=0,0 \leq \mathrm{k}<\infty$ be fixed and let $\mathrm{f}(\mathrm{z}) \in \mathrm{k}-\mathrm{US}_{\mathrm{q}}(\lambda, \mathrm{A}, \mathrm{B}, \gamma, \mathrm{t})$ and is of the form (1) then for a complex number $\mu$

$$
\left|\mathrm{a}_{3}-\mu \mathrm{a}_{2}^{2}\right| \leq \begin{cases}\frac{(\mathrm{A}-\mathrm{B}) \mathrm{M}_{1}(\mathrm{k})}{2\left\{\mathrm{Q}-\mathrm{Su}_{3}\right\} \phi_{2}} \mid 2+\frac{2 \mathrm{E}(\mathrm{k})-(1+\mathrm{B}) \mathrm{M}_{1}(\mathrm{k})}{2}+\frac{(\mathrm{A}-\mathrm{B}) \mathrm{M}_{1}(\mathrm{k})}{2\left\{\mathrm{P}-\mathrm{Ru}_{2}\right\}}\left(\mathrm{Ru}_{2}-\frac{\mu\left\{\mathrm{Q}-\mathrm{Su}_{3}\right\} \phi_{2}}{\left\{\mathrm{P}-\mathrm{Ru}_{2}\right\}\left(\phi_{1}\right)^{2}}\right), & \left(\mu>\delta_{1}\right) \\ \frac{(\mathrm{A}-\mathrm{B}) \mathrm{M}_{1}(\mathrm{k})}{2\left\{\mathrm{Q}-\mathrm{Su}_{3}\right\} \phi_{2}} & \left(\delta_{1} \leq \mu \leq \delta_{2}\right) \\ \frac{(\mathrm{A}-\mathrm{B}) \mathrm{M}_{1}(\mathrm{k})}{2\left\{\mathrm{Q}-\mathrm{Su}_{3}\right\} \phi_{2}}\left[\frac{2 \mathrm{E}(\mathrm{k})-(1+\mathrm{B}) \mathrm{M}_{1}(\mathrm{k})}{2}+\frac{(\mathrm{A}-\mathrm{B}) \mathrm{M}_{1}(\mathrm{k})}{2\left\{\mathrm{P}-\mathrm{Ru}_{2}\right\}}\left(\mathrm{Ru}_{2}-\frac{\mu\left\{\mathrm{Q}-\mathrm{Su}_{3}\right\} \phi_{2}}{\left\{\mathrm{P}-\mathrm{Ru}_{2}\right\}\left(\phi_{1}\right)^{2}}\right)\right] & \left(\mu<\delta_{2}\right)\end{cases}
$$


where

$$
\begin{aligned}
& \delta_{1}=\frac{\left(\phi_{1}\right)^{2}\left\{\mathrm{P}-\mathrm{Ru}_{2}\right\}}{\phi_{2}(\mathrm{~A}-\mathrm{B}) \mathrm{M}_{1}(\mathrm{k})\left\{\mathrm{Q}-\mathrm{Su}_{3}\right\}}\left\{\left\{2+2 \mathrm{E}(\mathrm{k})-(1+\mathrm{B}) \mathrm{M}_{1}(\mathrm{k})\right\}\left\{\mathrm{P}-\mathrm{Ru}_{2}\right\}+(\mathrm{A}-\mathrm{B}) \mathrm{M}_{1}(\mathrm{k}) \mathrm{Ru}_{2}\right\} \\
& \delta_{2}=\frac{\left(\phi_{1}\right)^{2}\left\{\mathrm{P}-\mathrm{Ru}_{2}\right\}}{\phi_{2}(\mathrm{~A}-\mathrm{B}) \mathrm{M}_{1}(\mathrm{k})\left\{\mathrm{Q}-\mathrm{Su}_{3}\right\}}\left\{\left\{2 \mathrm{E}(\mathrm{k})-(1+\mathrm{B}) \mathrm{M}_{1}(\mathrm{k})-2\right\}\left\{\mathrm{P}-\mathrm{Ru}_{2}\right\}+(\mathrm{A}-\mathrm{B}) \mathrm{M}_{1}(\mathrm{k}) \mathrm{Ru}_{2}\right\}
\end{aligned}
$$

and

$$
\mathrm{P}=(1+\gamma)[2, \mathrm{q}] \quad \mathrm{Q}=(1+\gamma[2, \mathrm{q}])[3, \mathrm{q}]
$$

$$
R=(1-\gamma)+\gamma[2, q] \quad S=(1-\gamma)+\gamma[3, q]
$$

and $\mathrm{M}_{1}(\mathrm{k}), \mathrm{E}(\mathrm{k})$ are defined in (3) and (4)

\section{Proof.}

If $\mathrm{f}(\mathrm{z}) \in \mathrm{k}-\mathrm{US}_{\mathrm{q}}(\lambda, \mathrm{A}, \mathrm{B}, \gamma, \mathrm{t})$ then it follows that

$$
\begin{aligned}
& \frac{(1-t)\left(\partial_{\mathrm{q}} R_{\mathrm{q}}^{\lambda} \mathrm{f}(\mathrm{z})+\gamma \mathrm{z}^{2} \partial_{\mathrm{q}}^{2} \mathrm{R}_{\mathrm{q}}^{\lambda} \mathrm{f}(\mathrm{z})\right)}{(1-\gamma)\left(\mathrm{R}_{\mathrm{q}}^{\lambda} \mathrm{f}(\mathrm{z})-\mathrm{R}_{\mathrm{q}}^{\lambda} \mathrm{f}(\mathrm{t} \mathrm{z})\right)+\gamma \mathrm{z}\left(\partial_{\mathrm{q}} \mathrm{R}_{\mathrm{q}}^{\lambda} \mathrm{f}(\mathrm{z})-\mathrm{t} \partial_{\mathrm{q}} \mathrm{R}_{\mathrm{q}}^{\lambda} \mathrm{f}(\mathrm{t} \mathrm{z})\right)} \prec \mathrm{q}_{\mathrm{k}}(\mathrm{z}) \\
& =1+\frac{(\mathrm{A}-\mathrm{B})}{2} \mathbf{M}_{1}(\mathrm{k}) \mathrm{z}+\frac{\left\{2 \mathrm{E}(\mathrm{k})-(1+\mathrm{B}) \mathrm{M}_{1}(\mathrm{k})\right\}(\mathrm{A}-\mathrm{B})}{4} \mathbf{M}_{1}(\mathrm{k}) \mathrm{z}^{2}+\cdots
\end{aligned}
$$

Now by the definition of subordination there exists a function $\omega$ analytic in $D$ with $\omega(0)=0$ and $|\omega(\mathrm{z})|<1$ such that

$$
\begin{aligned}
& \frac{(1-t)\left(z_{\mathrm{q}} R_{\mathrm{q}}^{\lambda} \mathrm{f}(\mathrm{z})+\gamma \mathrm{z}^{2} \partial_{\mathrm{q}}^{2} \mathrm{R}_{\mathrm{q}}^{\lambda} \mathrm{f}(\mathrm{z})\right)}{(1-\gamma)\left(\mathrm{R}_{\mathrm{q}}^{\lambda} \mathrm{f}(\mathrm{z})-\mathrm{R}_{\mathrm{q}}^{\lambda} \mathrm{f}(\mathrm{t} \mathrm{z})\right)+\gamma \mathrm{z}\left(\partial_{\mathrm{q}} \mathrm{R}_{\mathrm{q}}^{\lambda} \mathrm{f}(\mathrm{z})-\mathrm{t} \partial_{\mathrm{q}} \mathrm{R}_{\mathrm{q}}^{\lambda} \mathrm{f}(\mathrm{tz})\right)} \\
& =1+\frac{(\mathrm{A}-\mathrm{B})}{2} \mathbf{M}_{1}(\mathrm{k}) \omega(\mathrm{z})+\frac{\left\{2 \mathrm{E}(\mathrm{k})-(1+\mathrm{B}) \mathrm{M}_{1}(\mathrm{k})\right\}(\mathrm{A}-\mathrm{B})}{4} \mathbf{M}_{1}(\mathrm{k}) \omega^{2}(\mathrm{z})+\cdots
\end{aligned}
$$

Now from lemma 8, equation (23) and equation (24), We have

and

$$
\mathrm{a}_{2}=\frac{(\mathrm{A}-\mathrm{B}) \mathrm{M}_{1}(\mathrm{k}) \mathrm{c}_{1}}{2\left[\mathrm{P}-\mathrm{Ru}_{2}\right] \phi_{1}}
$$

$$
\begin{gathered}
\mathrm{a}_{3}=\frac{(\mathrm{A}-\mathrm{B}) \mathrm{M}_{1}(\mathrm{k})}{2\left[\mathrm{Q}-\mathrm{Su}_{3}\right] \phi_{2}}\left\{\mathrm{c}_{2}+\left(\frac{\left[2 \mathrm{E}(\mathrm{k})-(1+\mathrm{B}) \mathrm{M}_{1}(\mathrm{k})\right]}{2}+\frac{\mathrm{Ru}_{2}(\mathrm{~A}-\mathrm{B}) \mathrm{M}_{1}(\mathrm{k})}{2\left[\mathrm{P}-\mathrm{Ru}_{2}\right]}\right) \mathrm{c}_{1}^{2}\right\} \\
\left|\mathrm{a}_{3}-\mu \mathrm{a}_{2}^{2}\right|=\frac{(\mathrm{A}-\mathrm{B}) \mathrm{M}_{1}(\mathrm{k})}{2\left\{\mathrm{Q}-\mathrm{Su}_{3}\right\} \phi_{2}}\left|\mathrm{c}_{2}+\left\{\frac{2 \mathrm{E}(\mathrm{K})-(1+\mathrm{B}) \mathrm{M}_{1}(\mathrm{k})}{2}+\frac{(\mathrm{A}-\mathrm{B}) \mathrm{M}_{1}(\mathrm{k})}{2\left\{\mathrm{P}-\mathrm{Ru}_{2}\right\}}\left(\mathrm{Ru}_{2}-\frac{\mu\left\{\mathrm{Q}-\mathrm{Su}_{3}\right\} \phi_{2}}{\left\{\mathrm{P}-\mathrm{Ru}_{2}\right\}\left(\phi_{1}\right)^{2}}\right)\right\} \mathrm{c}_{1}^{2}\right|
\end{gathered}
$$

which gives

$$
\left|\mathrm{a}_{3}-\mu \mathrm{a}_{2}^{2}\right|=\frac{(\mathrm{A}-\mathrm{B}) \mathrm{M}_{1}(\mathrm{k})}{2\left\{\mathrm{Q}-\mathrm{Su}_{3}\right\} \phi_{2}}\left|\mathrm{c}_{2}-\mathrm{c}_{1}^{2}+\left\{1+\frac{2 \mathrm{E}(\mathrm{K})-(1+\mathrm{B}) \mathrm{M}_{1}(\mathrm{k})}{2}+\frac{(\mathrm{A}-\mathrm{B}) \mathrm{M}_{1}(\mathrm{k})}{2\left\{\mathrm{P}-\mathrm{Ru}_{2}\right\}}\left(\mathrm{Ru}_{2}-\frac{\mu\left\{\mathrm{Q}-\mathrm{Su}_{3}\right\} \phi_{2}}{\left\{\mathrm{P}-\mathrm{Ru}_{2}\right\}\left(\phi_{1}\right)^{2}}\right)\right\} \mathrm{c}_{1}^{2}\right|,
$$

Suppose that $\mu>\delta_{1}$ then using the estimates $\left|c_{2}-c_{1}^{2}\right| \leq 1$ from lemma 8 and the well known estimate $\left|c_{1}\right| \leq 1$ of the Schewarz lemma, we obtain

$$
\left|\mathrm{a}_{3}-\mu \mathrm{a}_{2}^{2}\right| \leq \frac{(\mathrm{A}-\mathrm{B}) \mathrm{M}_{1}(\mathrm{k})}{2\left\{\mathrm{Q}-\mathrm{Su}_{3}\right\} \phi_{2}} \mid 2+\frac{2 \mathrm{E}(\mathrm{k})-(1+\mathrm{B}) \mathrm{M}_{1}(\mathrm{k})}{2}+\frac{(\mathrm{A}-\mathrm{B}) \mathrm{M}_{1}(\mathrm{k})}{2\left\{\mathrm{P}-\mathrm{Ru}_{2}\right\}}\left(\mathrm{Ru}_{2}-\frac{\mu\left\{\mathrm{Q}-\mathrm{Su}_{3}\right\} \phi_{2}}{\left\{\mathrm{P}-\mathrm{Ru}_{2}\right\}\left(\phi_{1}\right)^{2}}\right),
$$

The inequality (27) is our required assertion (20) for $\mu>\delta_{1}$ on other hand if $\mu<\delta_{2}$ then (25) gives,

$$
\left|\mathrm{a}_{3}-\mu \mathrm{a}_{2}^{2}\right| \leq \frac{(\mathrm{A}-\mathrm{B}) \mathrm{M}_{1}(\mathrm{k})}{2\left\{\mathrm{Q}-\mathrm{Su}_{3}\right\} \phi_{2}}\left[\left|\mathrm{c}_{2}\right|+\left\{\frac{2 \mathrm{E}(\mathrm{k})-(1+\mathrm{B}) \mathrm{M}_{1}(\mathrm{k})}{2}+\frac{(\mathrm{A}-\mathrm{B}) \mathrm{M}_{1}(\mathrm{k})}{2\left\{\mathrm{P}-\mathrm{Ru}_{2}\right\}}\left(\mathrm{Ru}_{2}-\frac{\mu\left\{\mathrm{Q}-\mathrm{Su}_{3}\right\} \phi_{2}}{\left\{\mathrm{P}-\mathrm{Ru}_{2}\right\}\left(\phi_{1}\right)^{2}}\right)\right\}\left|\mathrm{c}_{1}^{2}\right|\right] \text {, }
$$


Applying the estimates $\left|c_{2}\right| \leq 1-\left|c_{1}\right|^{2}$ of lemma 8 and $\left|c_{1}\right| \leq 1$, We have

$$
\left|\mathrm{a}_{3}-\mu \mathrm{a}_{2}^{2}\right| \leq \frac{(\mathrm{A}-\mathrm{B}) \mathrm{M}_{1}(\mathrm{k})}{2\left\{\mathrm{Q}-\mathrm{Su}_{3}\right\} \phi_{2}}\left[\frac{2 \mathrm{E}(\mathrm{K})-(1+\mathrm{B}) \mathrm{M}_{1}(\mathrm{k})}{2}+\frac{(\mathrm{A}-\mathrm{B}) \mathrm{M}_{1}(\mathrm{k})}{2\left\{\mathrm{P}-\mathrm{Ru}_{2}\right\}}\left(\mathrm{Ru}_{2}-\frac{\mu\left\{\mathrm{Q}-\mathrm{Su}_{3}\right\} \phi_{2}}{\left\{\mathrm{P}-\mathrm{Ru}_{2}\right\}\left(\phi_{1}\right)^{2}}\right)\right]
$$

This is last inequality in (20). Finally if $\delta_{1}<\mu<\delta_{2}$ then

$$
\left|\frac{2 \mathrm{E}(\mathrm{k})-(1+\mathrm{B}) \mathrm{M}_{1}(\mathrm{k})}{2}+\frac{(\mathrm{A}-\mathrm{B}) \mathrm{M}_{1}(\mathrm{k})}{2\left\{\mathrm{P}-\mathrm{Ru}_{2}\right\}}\left(\mathrm{Ru}_{2}-\frac{\mu\left\{\mathrm{Q}-\mathrm{Su}_{3}\right\} \phi_{2}}{\left\{\mathrm{P}-\mathrm{Ru}_{2}\right\}\left(\phi_{1}\right)^{2}}\right)\right| \leq 1
$$

Therefore (25) yields

$$
\begin{gathered}
\left|\mathrm{a}_{3}-\mu \mathrm{a}_{2}^{2}\right| \leq \frac{(\mathrm{A}-\mathrm{B}) \mathrm{M}_{1}(\mathrm{k})}{2\left\{\mathrm{Q}-\mathrm{Su}_{3}\right\} \phi_{2}}\left\{\left|\mathrm{c}_{2}\right|+\left|\mathrm{c}_{1}\right|^{2}\right\} \\
\left|\mathrm{a}_{3}-\mu \mathrm{a}_{2}^{2}\right| \leq \frac{(\mathrm{A}-\mathrm{B}) \mathrm{M}_{1}(\mathrm{k})}{2\left\{\mathrm{Q}-\mathrm{Su}_{3}\right\} \phi_{2}}\left\{1-\left|\mathrm{c}_{2}\right|+\left|\mathrm{c}_{1}\right|^{2}\right\} \\
\left|\mathrm{a}_{3}-\mu \mathrm{a}_{2}^{2}\right| \leq \frac{(\mathrm{A}-\mathrm{B}) \mathrm{M}_{1}(\mathrm{k})}{2\left\{\mathrm{Q}-\mathrm{Su}_{3}\right\} \phi_{2}}
\end{gathered}
$$

We get the middle inequality in(20). This completes the proof.

\section{Theorem 17.}

Let $\leq \mathrm{k}<\infty,-1 \leq \mathrm{B}<\mathrm{A} \leq 1$ and $\mathrm{t}=0$ be fixed and let $\mathrm{f}(\mathrm{z}) \in \mathrm{k}-\mathrm{US}_{\mathrm{q}}(\lambda, \mathrm{A}, \mathrm{B}, \gamma, \mathrm{t})$ and is of the form (1). Then for a complex number $\mu$

where $\mathrm{v}$ is given by (31)

$$
\left|\mathrm{a}_{3}-\mu \mathrm{a}_{2}^{2}\right| \leq \frac{(\mathrm{A}-\mathrm{B})\left|\mathrm{M}_{1}(\mathrm{k})\right|}{\left\{\mathrm{Q}-\mathrm{Su}_{3}\right\} \phi_{2}} \max \{1,|2 v-1|\}
$$

Proof.

From (25) we have

$$
\begin{aligned}
\mid \mathrm{a}_{3}- & \mu \mathrm{a}_{2}^{2}\left|=\frac{(\mathrm{A}-\mathrm{B}) \mathrm{M}_{1}(\mathrm{k})}{2\left\{\mathrm{Q}-\mathrm{Su}_{3}\right\} \phi_{2}}\right| \mathrm{c}_{2}-\left\{\frac{(1+\mathrm{B}) \mathrm{M}_{1}(\mathrm{k})-2 \mathrm{E}(\mathrm{k})}{2}-\frac{(\mathrm{A}-\mathrm{B}) \mathrm{M}_{1}(\mathrm{k})}{2\left\{\mathrm{P}-\mathrm{Ru}_{2}\right\}}\left(\mathrm{Ru}_{2}-\frac{\mu\left\{\mathrm{Q}-\mathrm{Su}_{3}\right\} \phi_{2}}{\left\{\mathrm{P}-\mathrm{Ru}_{2}\right\}\left(\phi_{1}\right)^{2}}\right)\right\} \mathrm{c}_{1}^{2} \mid, \\
& =\frac{(\mathrm{A}-\mathrm{B}) \mathrm{M}_{1}(\mathrm{k})}{2\left\{\mathrm{Q}-\mathrm{Su}_{3}\right\} \phi_{2}}\left|\mathrm{c}_{2}-\mathrm{vc}_{1}^{2}\right|
\end{aligned}
$$

where

$$
v=\frac{(1+\mathrm{B}) \mathrm{M}_{1}(\mathrm{k})-2 \mathrm{E}(\mathrm{k})}{2}-\frac{(\mathrm{A}-\mathrm{B}) \mathrm{M}_{1}(\mathrm{k})}{2\left\{\mathrm{P}-\mathrm{Ru}_{2}\right\}}\left(\mathrm{Ru}_{2}-\frac{\mu\left\{\mathrm{Q}-\mathrm{Su}_{3}\right\} \phi_{2}}{\left\{\mathrm{P}-\mathrm{Ru}_{2}\right\}\left(\phi_{1}\right)^{2}}\right)
$$

Applying the lemma 7 on the equation (30), we obtain the required result.

\section{References}

[1] N.I. Ahiezer, "Elements of theory of elliptic functions", Moscow, 1970.

[2] S. Hussain, S. Khan, M.A. Zaighum and M.Darus, "Certain subclass of analytic functions related with conic domains and associated with salagean q-differential operator", AIMS Math.,vol.2, no. 4, pp. 622634, 2017.

[3] S.Hussain, S. Khan, M.A. Zaighum, M.Darus and Z.Shareef, "Coefficient Bounds for Certain Subclass of Biunivalent Functions Associated with Rucheweyh q-differential Operator", J. Complex Anal., 2017 (2017). 
[4] W. Janowski, "Some extremal problems for certain families of analytic functions", Ann. Polon.Math., vol. 28, pp. 297-326, 1973.

[5] S. Kanas and A.Wisniowska, "Conic regions and k-uniform convexity", J. Comput. Appl. Math.,vol. 105, pp. 327-336, 1999.

[6] S. Kanas and A. Wisniowska, "Conic domains and starlike functions", Rev. RoumaineMath.Pures Appl., vol. 45, pp. 647-657, 2000.

[7] S.Kanas and D. Raducanu, "Some classes of analytic functions related to conic domains", Math.slovaca, vol. 64, no. 5, pp. 1183-1196, 2014.

[8] F.R. Keogh and E.P. Merkes, "A coefficient inequality for certain classes of analytic functions", Proc. Amer. Math. Soc., vol. 20, pp. 8-12, 1969.

[9] N. Khan, B. Khan, Q.Z. Ahmad and S.Ahmad, "Some Convolution properties of Multivalent Analytic Functions",AIMS Math., vol. 2, no. 2, pp. 260-268, 2017.

[10] W. Ma and D. Minda, A unified treatment of some special classes of univalent functions. In: Proc. of the Conference on Complex Analysis (Tianjin), 1992 (Z. Li, F.Y.Ren, L.Yang, S.Y. Zhang,eds.), Conf. Proc.Lecture Notes Anal., Int. Press, Massachusetts, vol 1, pp. 157-169, 1994.

[11] K.I. Noor and S.N. Malik, "On coefficient inequalities of functions associated with conic domains", Comput. Math. Appl., vol. 62, pp. 2209-2217, 2011.

[12] K.I. Noor, J. Sokol and Q.Z. Ahmad, "Applications of conic type regions to subclasses of meromorphicunivalent functions with respect to symmetric points", Rev. R. Acad. Cienc. Exactas Fs.Nat., Ser. A Mat.,vol. 111, pp. 947C958, 2017.

[13] K.I. Noor, J. Sokol and Q.Z. Ahmad, "Applications of the differential operator to a class of meromorphic univalent functions",J. Egyptian Math. Soc.,vol. 24, no. 2, pp. 181-186, 2016.

[14] M. Nunokawa, S. Hussain, N.Khan and Q.Z. Ahmad, "A subclass of analytic functions related with conic domain", J. Clas. Anal.,vol. 9, pp. 137-149, 2016.

[15] W.Rogosinski, "On the coefficient of subordinate functions", Proc. Lond. Math. Soc., vol. 48, pp. 48-82, 1943.

[16] S.T. Rucheweyh, "New criteria for univalent functions", Proc. Amer. Math. Soc., vol. 49, pp. 109-115, 1975.

[17] H. Silverman, "Univalent functions with negative coefficient", Proc. Amer. Math. Soc., vol. 51, pp. 109116, 1975.

[18] S. Shams, S. R. Kulkarni and J.M. Jahangiri, "Classes of uniformly starlike and convex functions", Int. J. Math. Sci., vol. 55, pp. 2959-2961, 2004.

[19] S. Khaan, S. Hussain, M.S. Zhaighum and M. Mumtaz Khan, "New subclass of analytic functions in conical domain associated with Rucheweyh q-differential operator", IJAA, vol. 16, no. 2, pp. 239-253, 2018.

[20] G. Saravanan, Muthunagai. K, "Coefficient Estimates and Fekete- Szegö Inequality for a Subclass of Bi-Univalent Functions Defined by Symmetric Q-Derivative Operatorby Using Faber Polynomial Techniques", " Periodicals of Engineering and Natural Sciences, Vol.6, No.1, June 2018, pp. 241 250.

[21] N.P. Damodaran , SruthaKeerthi.B, "Coefficient bounds for a subclass of Sakaguchi type functions using Chebyshev Polynomial", " Periodicals of Engineering and Natural Sciences, Vol.6, No.1, June 2018, pp. 296-304.

[22] P. Murugabharathi, B. SruthaKeerthi , "Designing Filter for Certain Subclasses ofAnalytic Univalent Functions", " Periodicals of Engineering and Natural Sciences,Vol.6, No.1, June 2018, pp. 274 284

[23] Migdat I. Hodžić "Uncertainty Balance Principle"PERIODICALS OF ENGINEERING AND NATURAL SCIENCES Vol. 4 No. 2 (2016).

[24] Narayanan Venkateswaran "Efficient read monotonic data aggregation across shards on the cloud" Periodicals of Engineering and Natural Sciences Vol.7, No.1, June 2019, pp.125-140. 